\title{
Micro Drilling Simulation of Ultra-Short Pulsed Laser Ablation of Glass
}

\author{
Fumitaka Motomura \\ Division of System Science, Nagasaki University \\ 1-14 Bunkyo-machi, Nagasaki-shi, Nagasaki 852-8521, Japan \\ E-mail: motomura@nagasaki-u.ac.jp \\ [Received January 26, 2015; accepted June 12, 2015]
}

\begin{abstract}
The purpose of this study is to estimate ablated crater depth with sufficient numerical accuracy when multishot channels of ultra-short pulsed laser are executed for micro drilling processes on thin glass plates. In this analytical model, the plasma model, in which the free electron density and the complex dielectric function of the Lorentz model are evaluated, is applied to estimate the ablated regions and the regions damaged by laser ablation when glass is considered to be a dielectric material. The absorption coefficient and the threshold fluence are important parameters in the evaluation of the ablated crater depth and ablation rate. The parameters obtained in this numerical analysis are in agreement with the experimental results and are computed quantitatively to several laser irradiation conditions. The experimental results and analysis results are examined for multi-shot channels. In an experiment involving laser ablation using multi-shot laser beams, ablation rates for the initial shot are lower than subsequent ablation rates. The effectiveness of the modified absorption coefficient and modified threshold fluence for initial shots is confirmed for the reduction of ablation rate.
\end{abstract}

Keywords: ultra-short pulsed laser, laser ablation, micro drilling, threshold fluence, absorption coefficient

\section{Introduction}

The spread of smart phone and tablet PCs has created a need for a high-precision method of processing the glass used for their display. The trend in the display industry is towards thinner and lighter devices. With the ongoing development of laser technology, laser ablation with ultra-short pulsed laser for glass cutting is increasingly being recognized as a promising solution. This is because the laser ablation process is superior as a laser scribe and to contact cutting tool for drilling or gouging processes on thin glass plates. The laser ablation process using laser pulses ablates material and drills through glass without subjecting the material to thermal or mechanical stress. Because laser ablation is an ultra-high-speed phenomenon, in situ measurement of the consecutive time variation of the surface shape removed by each shot of ultra-short pulsed laser is impossible. After using trial and error in preliminary experiments, processing conditions are decided. In this study, numerical simulations of laser ablation are executed using ultra-short pulsed laser for glass micro drilling.

\section{Analytical Model}

\subsection{Electric Fields Distribution Formed by Laser Beam Which Spreads to Inside of Transparent Dielectrics}

When glass is considered to be a dielectric material, it is necessary to analyze its electric field by means of the non-linear absorption of the pulsed laser beam in order to estimate the distribution in time and space of the laser beam intensity to propagate the material inside. The beam propagation method is a numerical technique that is used frequently when the distribution in time and space of laser beam intensity is estimated.

First, the deviation of the paraxial Helmholtz equation is necessary for the electric field analysis. The equation showing the two-dimensional TE wave (transverse electric field) is the same as the two-dimensional scalar wave equation, a partial differential equation of an elliptical type. We assume that the electric field propagates in the $z$ direction and that the structural change to the propagation direction of objects is smooth. The equation can become a partial differential equation of a parabolic type by approximating the electric field of TE mode $E_{y}(x, z)$ by using the product of the function $\exp \left(\mathrm{i} k_{0} n_{0} z\right)$ rapidly changed to wavelength order in the $z$ direction, and the function $F(x, z)$ slowly changes to the $z$ direction, as shown in Eq. (1).

$$
E_{y}(x, z)=F(x, z) \exp \left(\mathrm{i} k_{0} n_{0} z\right) \quad \ldots . . .
$$

where $k_{0}=2 \pi / \lambda, \lambda$ is the laser wavelength, $n_{0}$ is the refractive index of transparent dielectrics, and $F(x, z)$ is the envelope of the electric field inside of the target materials. Inserting Eq. (1) into the Helmholtz equation and assuming slowly varying envelope approximation in the $z$ direction, we get the paraxial Helmholtz equation.

$$
2 \mathrm{i} k_{0} n_{0} \frac{\partial F}{\partial z}=-\left(\frac{\partial^{2}}{\partial x^{2}}+\frac{\partial^{2}}{\partial z^{2}}\right) F-k_{0}^{2}\left\{\varepsilon-n_{0}^{2}\right\} F
$$


where $\varepsilon$ is the dielectric function. Eq. (2) is developed using the finite differential type of beam propagation method, and the envelope of the electric field formed by laser absorption is obtained.

Next, with non-linear absorption of laser beam intensity induced by radiation of the ultra-short pulsed laser, it is expected that the optical properties of materials change in quality because sudden phase conversion from solid to plasma occurs for the most part. As there are two complex dielectric functions, the Drude model or Larentz model is selected [1-5]. In this analysis, in order to express the interaction of laser absorption and target materials, the classic Lorentz model for the bound electron is adapted to the dielectric function. The complex dielectric function $\varepsilon$ is given by $[1,2]$.

$$
\begin{array}{r}
\varepsilon=\{1+ \\
\left.+\frac{\rho_{B} e^{2}}{\varepsilon_{0} m_{e}} \frac{\omega_{0}^{2}-\omega^{2}}{\left(\omega_{0}^{2}-\omega^{2}\right)^{2}+\chi^{2} \omega^{2}}\right\} \\
+\mathrm{i} \frac{\rho_{B} e^{2}}{\varepsilon_{0} m_{e}} \frac{\chi \omega}{\left(\omega_{0}^{2}-\omega^{2}\right)^{2}+\chi^{2} \omega^{2}}
\end{array}
$$

where $\omega, m_{e}, e, \varepsilon_{0}, \rho_{B}$, and $\omega_{0}$ are the laser angular frequency, the effective mass of a quasi-free electron, the electron charge, the vacuum dielectric permittivity, the bound electron density, and the frequency of the harmonic oscillators, respectively. $\chi$ is the damping constant of the harmonic oscillators, which means the energy loss in laser absorption. The dumping constant $\chi$ is given by [2].

$$
\chi=\chi_{\max } \frac{\rho_{\max }}{\rho_{\max }+\beta \rho_{\text {crit }}}
$$

In Eq. (4), $\rho_{\max }$ is the maximum free-electron density within the pulse duration, $\beta$ is a free parameter in the model that can be selected to fit the ablated crater depth in single-shot experiments and $\rho_{\text {crit }}$ is the critical electron density. $\rho_{\text {crit }}=\omega^{2} m_{e} \varepsilon_{0} / e^{2}$ is generally used. $\chi_{\max }$ is fixed once parameters $\rho_{B}$ and $\omega_{0}$ are chosen: it is the value of the damping for which the absorption coefficient is the maximum. The absorption coefficient of target materials $\alpha_{\text {eff }}$ is given by [3-5].

$$
\alpha_{\mathrm{eff}}=k_{0} \sqrt{2}\left[-\operatorname{Re}(\varepsilon)+\sqrt{\operatorname{Re}(\varepsilon)^{2}+\operatorname{Im}(\varepsilon)^{2}}\right]^{1 / 2}
$$

The response of transparent dielectrics to the incident radiation is determined by the complex dielectric function. In addition, estimated formulas of the ablated crater depth using the absorption coefficient are often used. The BeerLambert law may be applied as an estimated formula of ablated crater depth obtained in the experiment, and the increment of ablated crater depth per shot within the multi-shot of pulsed laser $h_{a}$, hereinafter referred to as the ablation rate is given by $[6,7]$.

$$
h_{a}=\alpha_{\mathrm{eff}}^{-1} \ln \left(\frac{F_{0}}{F_{\mathrm{th}}}\right)=\alpha_{\mathrm{eff}}^{-1} \ln \left(\frac{Q}{A W_{0}^{2} F_{\mathrm{th}}}\right) .
$$

where $F_{\text {th }}, F_{0}, Q, W_{0}$, and $A$ are the threshold fluence, the laser fluence, the laser energy, the beam waist of the half width at half maximum on the spatial Gaussian profile,

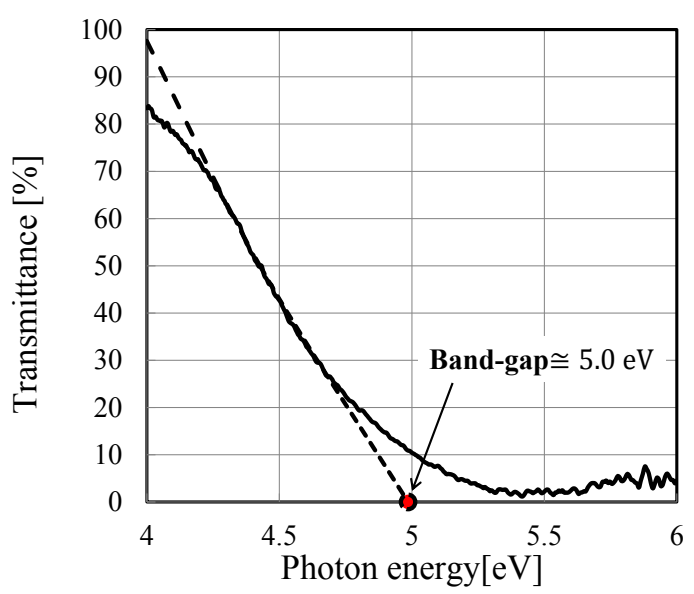

Fig. 1. Experimental estimation of band-gap for Corning non-strengthened gorilla glass.

and a constant which means an effective irradiated laser beam area expressed by the beam waist, respectively.

\subsection{Temporal Evolution of Free Electron Density and Calculation of Threshold Fluence Using Nonlinear Laser Absorption and Interaction of Laser Beam and Material}

Affected by the laser field, the electrons in the valence band are excited to the conduction band by multi-photon ionization (MPI) and then are heated to higher energy levels to initialize cascade ionization by means of inverse Bremsstrahlung absorption. Those ionizations act to increment electron density while the decrement of electron density will be described by radiations such as the recombination and diffusion processes. The temporal evolution of electron density $\rho$ in the conduction band is calculated using a rate equation of the generic form [8].

$$
\frac{\partial \rho}{\partial t}=\sigma I(t)^{k}+\eta_{c} I(t) \rho-\eta_{\mathrm{rec}} \rho^{2}-\eta_{\mathrm{diff}} \rho \quad .
$$

where the terms on the right side are the MPI rate, the cascade ionization rate, and the recombination rate and diffusion rate, respectively. $\sigma, \eta_{c}, \eta_{\text {rec }}$ and $\eta_{\text {diff }}$ in Eq. (7) are given by references [1, 2, 8-10].

The influences of pulsed duration on the electron dynamics are elucidated by analyzing the temporal evolution of free-electron density. For the ranges of laser energy (from $45.0 \mu \mathrm{J}$ to $75.0 \mu \mathrm{J}$ ) used in our laser ablation experiments, the reasonable process of MPI confirmed the estimate of the Keldysh parameter [8,10-12]. MPI parameter $k$ of laser intensity is $k=\left\langle E_{g} / \hbar \omega+1\right\rangle$. $E_{g}$ and $\hbar$ are the band-gap of transparent materials and the reduced Planck constant, respectively. The glass plates used in experiments are Corning non-strengthened gorilla glass, and their band-gap obtained from the measurement of transmittance is about $5.0 \mathrm{eV}$, as shown in Fig. 1. Band-gap is extrapolated from a tangent line at an inflection point of transmittance that decreases sharply as the wavelength of incident light shortens. 
Table 1. Important parameters in numerical analysis and micro drilling experiment.

\begin{tabular}{|l|c|r|r|}
\hline \multicolumn{5}{|c|}{ In Laser Intensity } \\
\hline Wave length & $\lambda$ & 515 & {$[\mathrm{~nm}]$} \\
\hline Pulse duration & $t_{\mathrm{p}}$ & 6.0 & {$[\mathrm{ps}]$} \\
\hline Pulse repetition rate & & 10.0 & {$[\mathrm{kHz}]$} \\
\hline Laser energy & $Q$ & $45,60,75$ & {$[\mu \mathrm{J}]$} \\
\hline Beam waist & $W_{0}$ & $7.75,11.50,15.75$ & {$[\mu \mathrm{m}]$} \\
\hline \multicolumn{4}{|c|}{ In Complex Dielectric Function } \\
\hline Bound electron density & $\rho_{\mathrm{B}}$ & $1.46 \times 10^{23}$ & {$\left[\mathrm{~cm}^{-3}\right]$} \\
\hline $\begin{array}{l}\text { Frequency of } \\
\text { the harmonic oscillator }\end{array}$ & $\omega_{0}$ & 19.0 & {$\left[\mathrm{fs}^{-1}\right]$} \\
\hline $\begin{array}{l}\text { Critical free electron } \\
\text { density }\end{array}$ & $\rho_{\text {crit }}$ & $4.2 \times 10^{21}$ & {$\left[\mathrm{~cm}^{-3}\right]$} \\
\hline Fitting parameter & $\beta$ & 0.1 & \\
\hline $\begin{array}{l}\text { Maximum damping } \\
\text { constant }\end{array}$ & $\chi_{\text {max }}$ & $30,40,50$ & {$\left[\mathrm{fs}{ }^{-1}\right]$} \\
\hline \multicolumn{4}{|c|}{ In Rate Equation of Free Electron Density } \\
\hline Band gap & $E_{\mathrm{g}}$ & \multicolumn{3}{|c|}{5.0} & {$[\mathrm{eV}]$} \\
\hline Refractive index & $n_{0}$ & 1.5 & \\
\hline \multicolumn{4}{|c|}{ Constant paramters } \\
\hline Speed of light & $c$ & $2.99792458 \times 10^{8}$ & {$[\mathrm{~m} / \mathrm{s}]$} \\
\hline $\begin{array}{l}\text { Effective mass of a } \\
\text { quasi-free electron }\end{array}$ & $m_{\mathrm{e}}$ & $9.10938215 \times 10^{-31}$ & {$[\mathrm{~kg}]$} \\
\hline Electron charge & $e$ & $1.60217650 \times 10^{-19}$ & {$[\mathrm{C}]$} \\
\hline $\begin{array}{l}\text { Vacuum dielectric } \\
\text { permittivity }\end{array}$ & $\varepsilon$ & $8.85418782 \times 10^{-12}$ & {$[\mathrm{~F} / \mathrm{m}]$} \\
\hline Reduced Plank constant & $\hbar$ & $6.58264844 \times 10^{-16}$ & {$[\mathrm{eVs}]$} \\
\hline
\end{tabular}

The time variation of laser intensity in this analysis used the following version of the Gaussian profile for pulse duration $t_{p}$.

$$
\begin{aligned}
I(t) & =I_{0} \exp \left\{-4.0 \ln 2.0\left(\frac{t}{t_{p}}\right)^{2}\right\} \ldots . . \\
I_{0} & =\frac{Q}{\pi\left(1.0 \times 10^{-4}\right)^{2}} \\
& \times \frac{1}{\int_{-\infty}^{+\infty} \exp \left\{-4 \ln 2\left(\frac{t}{t_{p}}\right)^{2}\right\} d t}\left[\mathrm{~W} / \mathrm{cm}^{2}\right]
\end{aligned}
$$

where $t_{p}$ is defined as full-width half-maximum. The spatial laser intensity in this analysis uses the following version of the Gaussian profile for beam waist $W_{0}$ as well.

$$
\begin{aligned}
& I(x)=I_{1} \exp \left\{-0.693147\left(\frac{x}{W_{0}}\right)^{2}\right\} \ldots . \\
& I_{1}=I_{0} \frac{\left(1.0 \times 10^{-4}\right)^{2}}{\sum_{i=1}^{n=\infty} \frac{W_{0}^{2}}{0.693147} \ln \left(\frac{2 n}{2 i-1}\right)}\left[\mathrm{W} / \mathrm{cm}^{2}\right] .
\end{aligned}
$$

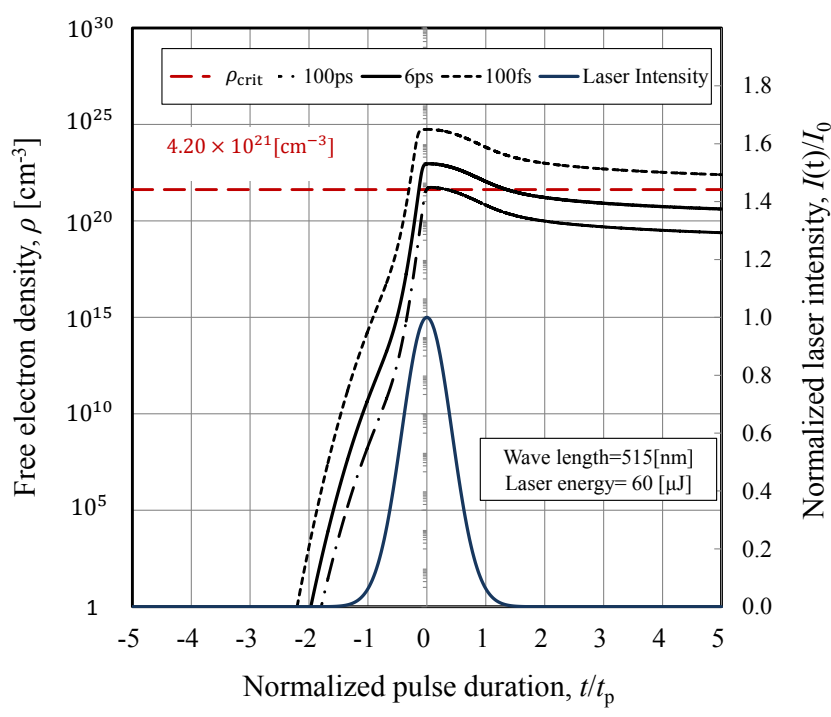

Fig. 2. Temporal evolution of free electron density during the laser pulse at green wavelength for several pulse durations and time variation of the normalized laser intensity.

where $W_{0}$ is defined as half-width half-maximum. Through standardization with an electric field, the spatial peak laser intensity $I_{1}$ is given by $I_{1}=c \varepsilon_{0} n(x, z)|F|^{2}$. The modified refractive index $(n(x, z))$ which changed when the ablated material is damaged is substituted into the standardization formula mentioned above. The refractive index is calculated from the real part of the complex dielectric function in Eq. (3) inside target material. The interaction between the optical constant and absorbed laser intensity is reflected after the second shot and subsequently in the multi-shot channels.

The parameters required for the numerical analysis and experimental conditions of the ultra-short pulsed laser frequency device are listed in Table 1. The pulse repetition rate used in the micro drilling experiment was $10 \mathrm{kHz}$. It was a sub-millisecond longer than that of the burst mode, or a period of several nanoseconds. Refer to [2] for the parameters related to the temporal evolution of free electron density.

Figure 2 shows the temporal evolution of the free electron density $\rho$ and time variation of the normalized laser intensity $I(t) / I_{0}$ to three pulse durations $t_{p}=0.1 \mathrm{ps}$, $6.0 \mathrm{ps}$, and $100.0 \mathrm{ps}$ when laser energy $Q$ is $60.0 \mu \mathrm{J}$. When the wavelength of incident radiation is $515 \mathrm{~nm}$, critical free electron density is $4.20 \times 10^{21} \mathrm{~cm}^{-3}$. As shown in Fig. 2, when the temporal laser intensity increases, just before reaching peak value, free electron density $\rho$ reaches the maximum value, but $\rho$ drops gradually while temporal laser intensity falls to zero. Since the contribution of the cascade ionization will become strong in addition to multi-photon ionization if laser intensity approaches peak value, a high level is maintained for some time. The effect of radiation attenuates electron density over a much longer period of time than it does pulse duration. The profiles of time evolution of electron density for some pulse durations have shown a similar tendency. Moreover, since peak values of laser intensity increase as 


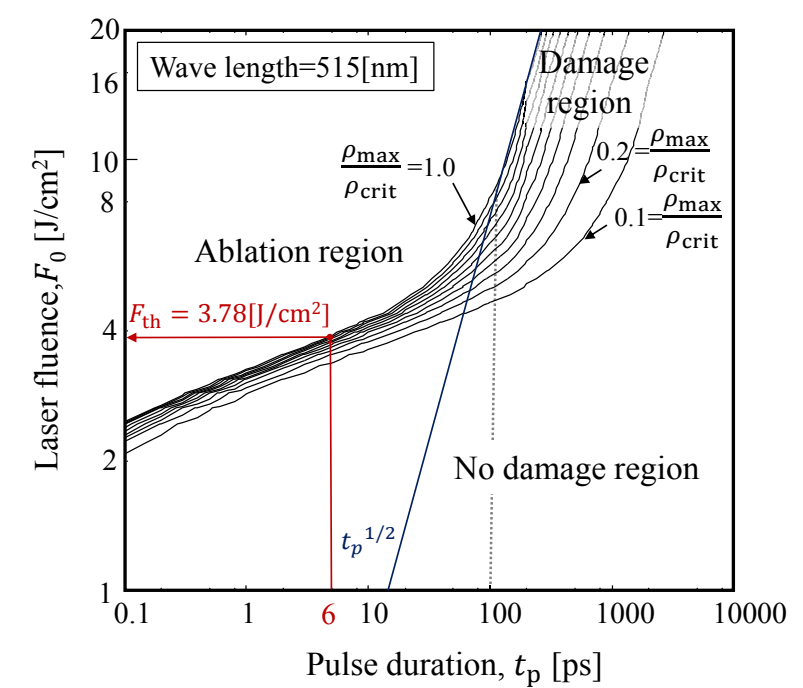

Fig. 3. Estimation of the threshold fluence and damaged region under various combinations of pulse duration and laser fluence.

short as pulse duration still remains constant laser energy, maximum free electron density increases. If maximum electron density $\rho_{\max }$ exceeds $\rho_{\text {crit }}$, ablation conditions are fulfilled. Ablation can be started with less laser energy when pulse duration is short.

Figure 3 shows the variation in the ablated region and the damaged region for several combinations of pulse duration and laser fluence. The range of laser fuluence $F_{0}$ was $1.0 \mathrm{~J} / \mathrm{cm}^{2}$ to $20.0 \mathrm{~J} / \mathrm{cm}^{2}$, and the range of pulse duration $t_{p}$ was $0.1 \mathrm{ps}$ to $10000 \mathrm{ps}$. Threshold fluence is the value of the point at which plasma arises, or when maximum free electron density exceeds critical free electron density and target materials are ablated. It is difficult to evaluate the region damaged in the experiment. In this analysis, maximum free electron density $\left(\rho_{\max } \mathrm{cm}^{-3}\right)$ set the damaged region as the region beyond $10 \%$ of critical free electron density $\left(\rho_{\text {crit }} \mathrm{cm}^{-3}\right)$. The damage region becomes large, so the ratio for $\rho_{\text {crit }}$ becomes small, and the ablation rate in subsequent laser pulses increases. When the lower limit of damage threshold in first-shot is a value of $10 \%$, the ablation rate in subsequent shot almost converges. Band-gap is $5.0 \mathrm{eV}$ and wave length is $515 \mathrm{~nm}$ in Fig. 3. When pulse duration became shorter than $100 \mathrm{ps,}$ the threshold fluence swerved from the line of $t_{p}^{0.5}$ [13]. The damaged region narrowed rapidly as it approached this pulse duration (100 ps), and it became almost constant while pulse duration was shorter than $10 \mathrm{ps}$. This is the reason why there is little damage, which is the advantage of ablation processing using ultra-short pulsed laser. Threshold fluence to the pulse duration $6 \mathrm{ps}$ used for experiments was computed using $3.78 \mathrm{~J} / \mathrm{cm}^{2}$.

\section{Results of Analytical}

\subsection{Laser Fluence Dependence of Absorption Co- efficient in Multi-Shot Channels}

Figure 4 shows the multi-shot ablation rate measurements of target materials with the picosecond pulsed laser

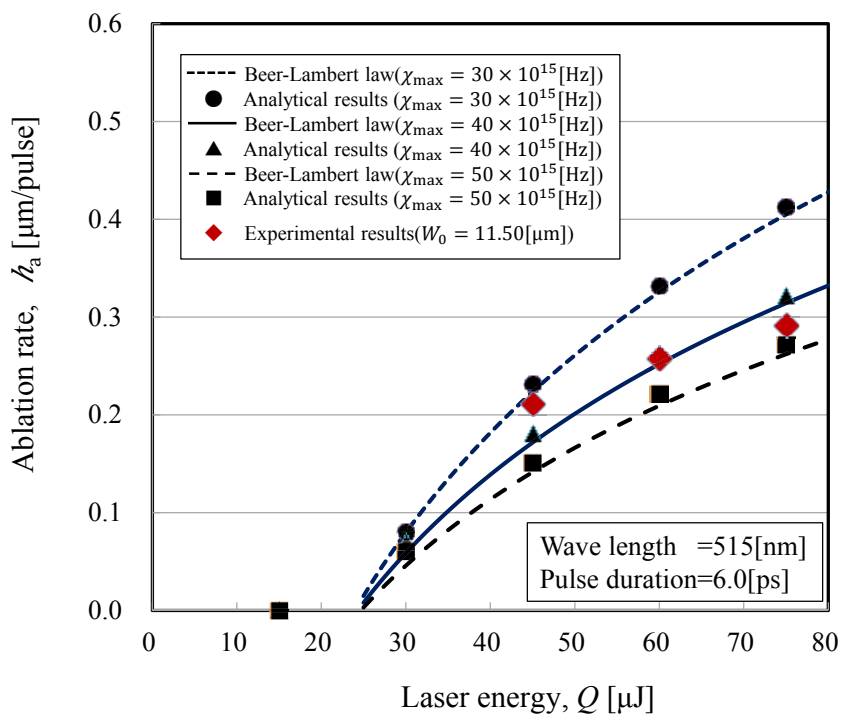

Fig. 4. The multi-shot ablation rate measurements of target materials with the picosecond pulsed laser and the singleshot ablation rate calculations of $\chi_{\max }$ related to the absorption coefficients.

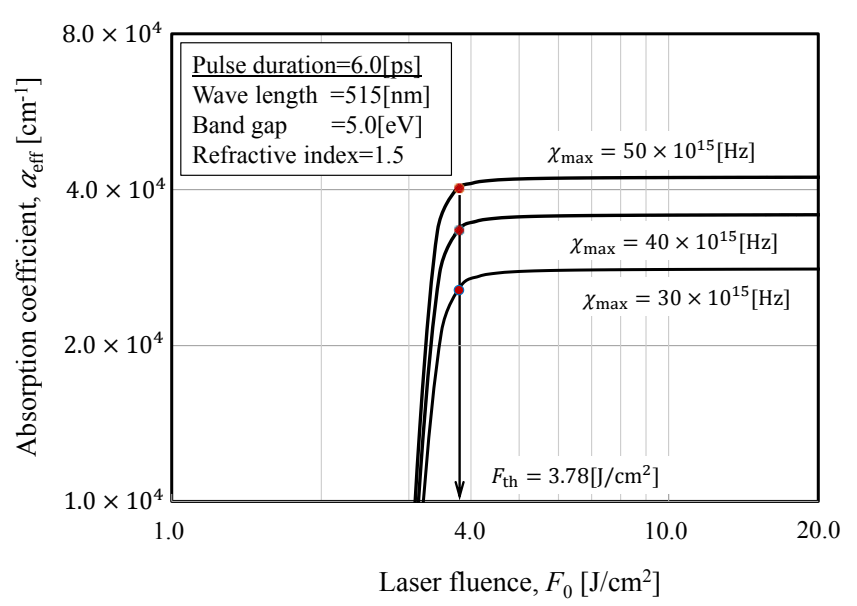

Fig. 5. Relationship between the absorption coefficient and the laser fluence obtained from $\chi_{\max }=30.0,40.0,50.0 \mathrm{fs}^{-1}$.

and the single-shot ablation rate calculations of $\chi_{\max }$ related to absorption coefficients. Multi-shot ablation rates obtained through experiments using picosecond pulsed laser increased as laser energy increased, as shown by the $\checkmark$ symbols in Fig. 4. Since there was no information relevant to the absorption coefficient of the target material, it was the first issue in the numerical analysis to search absorption coefficient which accorded with experimental results. The most influential parameter in calculating an absorption coefficient is $\chi_{\max }$, which is the maximum damping constant included in Eq. (4). The variation in the absorption coefficient to laser fluence for $\chi_{\max }$ is shown in Fig. 5. In Fig. 5, with the increase in $\chi_{\max }$, the convergence value of absorption coefficient increased, and it was unrelated to $\chi_{\max }$. However, it reached the convergence value immediately after exceeding the threshold of influence $\left(F_{\mathrm{th}}=3.78 \mathrm{~J} / \mathrm{cm}^{2}\right)$. In Fig. 4, the ablation rates obtained in the experimental results were held the abla- 


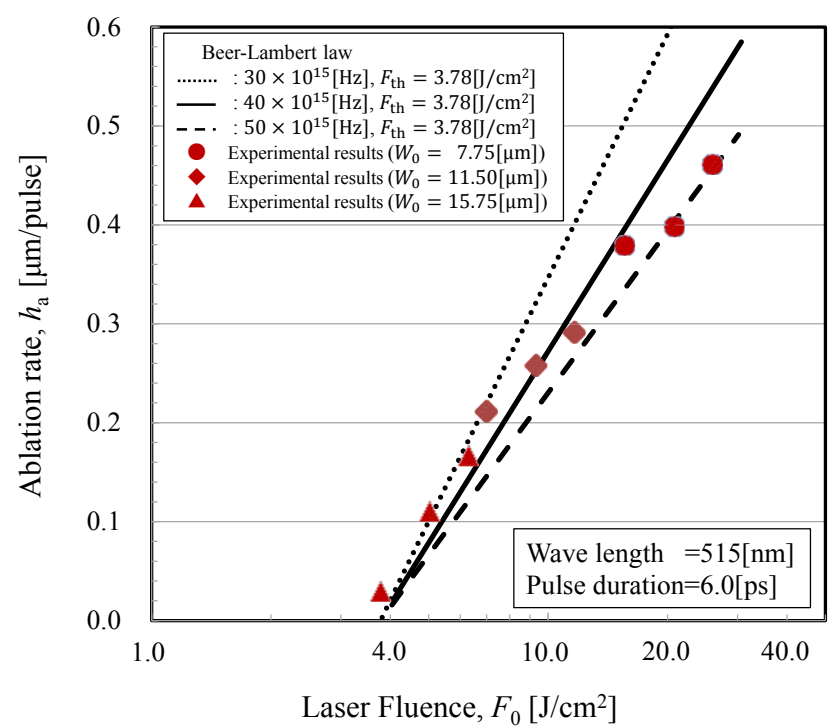

Fig. 6. The multi-shot ablation rate measurements obtained for several laser fluences with the picosecond pulsed laser and the single-shot ablation rate calculations based on the Beer-Lambert law.

tion rates obtained in the analysis results when $\chi_{\max }$ was set to the range from $30.0 \mathrm{fs}^{-1}$ to $50.0 \mathrm{fs}^{-1}$. In addition to the ablation rate obtained from our numerical model, the ablation rates based on the Beer-Lambert law of Eq. (6) are drawn as several different lines in Fig. 4. Analytical results of ablation rates in our numerical model as indicated with $\boldsymbol{\bullet}, \boldsymbol{\Delta}$, and $\boldsymbol{\square}$ symbols in Fig. 4, were in good agreement with the results of the Beer-Lambert law using absorption coefficients corresponding to each $\chi_{\max }$, which should be modified depending on laser energy. It was confirmed that numerical analysis in consideration of the laser fluence dependence of the absorption coefficient was necessary for an exact estimation of the ablation rate.

Figure 6 shows the variation in ablation rates with different laser fluences. These were obtained as experimental and analytical results when beam waists $W_{0}$ were $7.75 \mu \mathrm{m}, 11.50 \mu \mathrm{m}$, and $15.75 \mu \mathrm{m}$. The three lines in Fig. 6 are the ablation rates calculated using the BeerLambert law of Eq. (6), in which the absorption coefficients for $\chi_{\max }=30.0 \mathrm{fs}^{-1}, 40.0 \mathrm{fs}^{-1}, 50.0 \mathrm{fs}^{-1}$ were used. The ablation rates of experiments increased with increases in laser fluence; beam waists had no effect. The following tendencies are seen in the ablation rates in experiments. The ablation rates in experiments approached the ablation rate of $\chi_{\max }=30.0 \mathrm{fs}^{-1}$ at lower laser fluences, and the rate approached the ablation rate of $\chi_{\max }=50.0 \mathrm{fs}^{-1}$ at higher laser fluences.

In order to estimate the ablated crater depth, numerical analysis taking into consideration the increase in the absorption coefficient accompanying the increase in the laser fluence is required. According to Adela BenYakar [6, 7] and M. Lenzne [14], who conducted experiments on the reduction of ablated crater depth accompanying increases in laser fluence to multi-shot channels, in comparison with ablation experiments in vacuums and in air, reductions in ablated crater depth become remarkable in air. The environmental factors, such as the air resistance, and the chemical reaction in the air contact layer of the glass surface are mentioned as possible causes. The present analysis model cannot yet determine laser fluence dependence on the absorption coefficient analytically. Also, improvements for coping with the effects that environmental parameters have on ablation rates will be necessary in the future.

\subsection{Comparison of the Experimental Results and Analytical Results of the Depth of Ablated Crater in Multi-Shot Channels}

The correlation of the Beer-Lambert law, which is the law used in estimating the ablation rate, with our analysis model was confirmed. The Beer-Lambert law consists of an absorption coefficient and threshold fluence. Threshold fluence is the laser fluence when ablation occurs, and is a value peculiar to materials. The threshold fluence calculated by our analysis model $\left(3.78 \mathrm{~J} / \mathrm{cm}^{2}\right)$ accords with experimental results (external approximation: $3.69 \mathrm{~J} / \mathrm{cm}^{2}$ ) to a considerable degree, and the former can be applied enough for estimating threshold fluence as the ablation threshold. Therefore, it is supposed that the cause of the reduction in the ablation rate with the rise in laser fluence is the absorption coefficient dependence on laser fluence. The absorption coefficient that matches the ablation rate obtained through experimentation using the Beer-Lambert law is provided. The absorption coefficient including laser fluence dependence will be formulated in the next equation,

$$
\alpha_{\mathrm{eff}}=\alpha_{0}\left(1+\alpha_{1} \frac{F_{0}-F_{\mathrm{th}}}{F_{\mathrm{th}}}\right)
$$

The absorption coefficient obtained through experiments with a picosecond-pulsed laser is defined as a continuous function of laser fluence by using Eq. (12), and numerical analysis of any laser fluence is carried out effectively. In unknowns $\alpha_{0}$ and $\alpha_{1}$ in Eq. (12), two values to show through a least square approximation in Fig. 7 were provided. In the analytical model, it is necessary to set $\chi_{\max }$ for an initial constant, and $\chi_{\max }$, such as in Eq. (13), is derived using the absorption coefficient in Eq. (12).

$$
\chi_{\max }=1.0 \times 10^{16} \exp \left(3.83 \times 10^{-5} \alpha_{\text {eff }}\right)[\mathrm{Hz}] .
$$

The subsequent analysis results are calculated using Eq. (13).

Symbols $\boldsymbol{\bullet}, \boldsymbol{\nabla}$, and $\mathbf{\Delta}$ in Fig. 8 are experimental data showing ablated crater depth obtained after multi- shots up to five shots, when beam waists $W_{0}$ are $7.75,11.50$, $15.75 \mu \mathrm{m}$. In this experiment, the focus position of the ultra-short pulsed laser was fixed on the surface of the sample to be processed. The experimental results showed that the ablated crater depth after single-shot ablation was shallower than that in multi-shot ablation. It is thought that the ambient air and composition of the glass surface layer are related. In looking for causes for the reduced ablated crater depth after single-shot ablation, we focused on the rise in threshold fluence in the air contact 


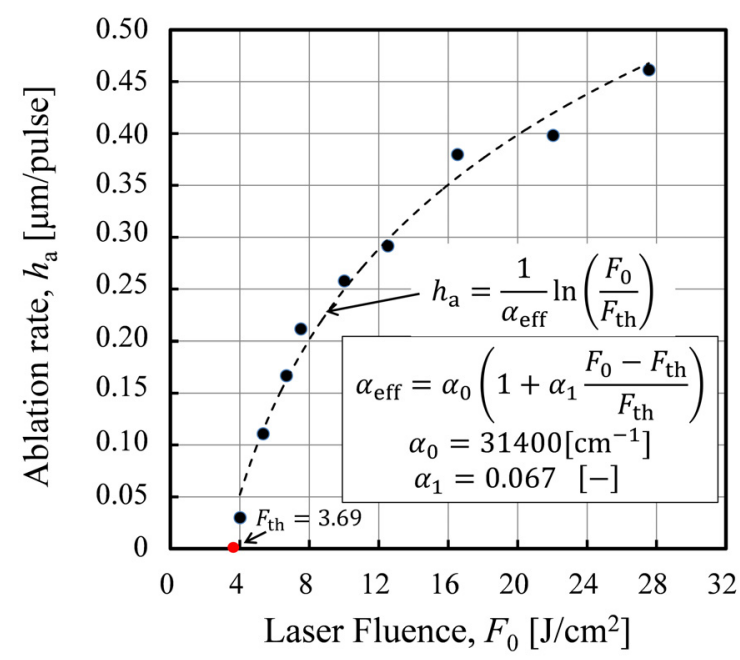

Fig. 7. Relationship between ablation rate and laser fluence in experimental results.

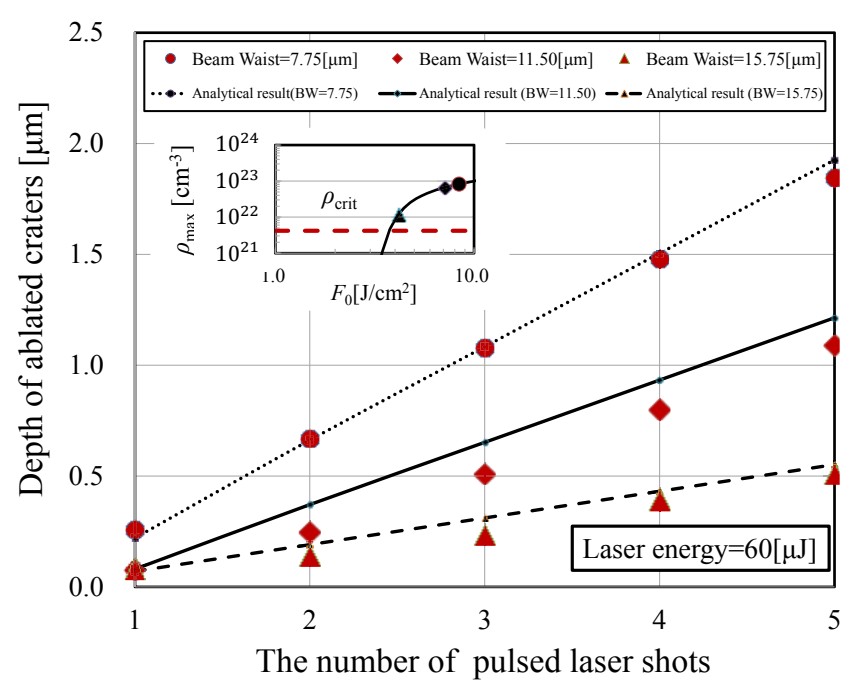

Fig. 8. Variation of the depth of ablated crater to the number of pulsed laser shots obtained by various beam waists.

layer of the glass surface. In order to correct threshold fluence for single-shot, the Beer-Lambert law in Eq. (6) is applied. Threshold fluences are computable by using ablated crater depths obtained through experiments, laser fluences used for experiments, and absorption coefficients obtained through analysis. The modified threshold fluences obtained for single-shot ablation were $F_{\text {th }}=8.40$, 7.10 , and $4.17 \mathrm{~J} / \mathrm{cm}^{2}$ to $Q=60.0 \mu \mathrm{J}$, and $W_{0}=7.75$, 11.50 , and $15.75 \mu \mathrm{m}$ respectively. Those threshold fluences are larger than threshold fluence $3.78 \mathrm{~J} / \mathrm{cm}^{2}$ used in multi-shot ablation. The rate of increase from threshold fluence in multi-shot ablation increased between 1.1 times to 2.2 times. The ratio with threshold fluences was so large that $W_{0}$ was small, and the reduction in ablated crater depth became remarkable. The three lines in Fig. 8 represent the analytical results obtained by modified threshold fluences in single-shot ablation and the original threshold fluence in subsequent shots. All ablated crater depths corresponded for single-shot ablation. On the other hand,

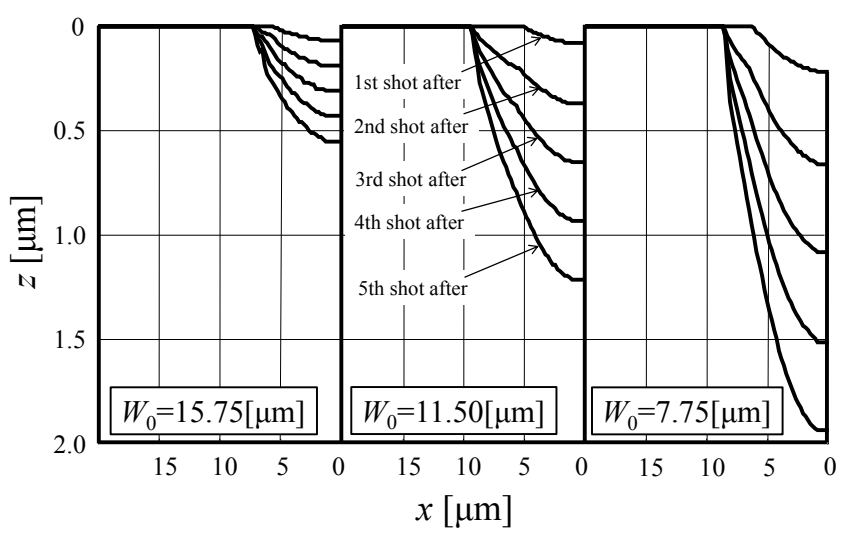

Fig. 9. Variation in the crater shape formed for every number of pulsed laser shot up to 5 times, obtained using various beam waists.

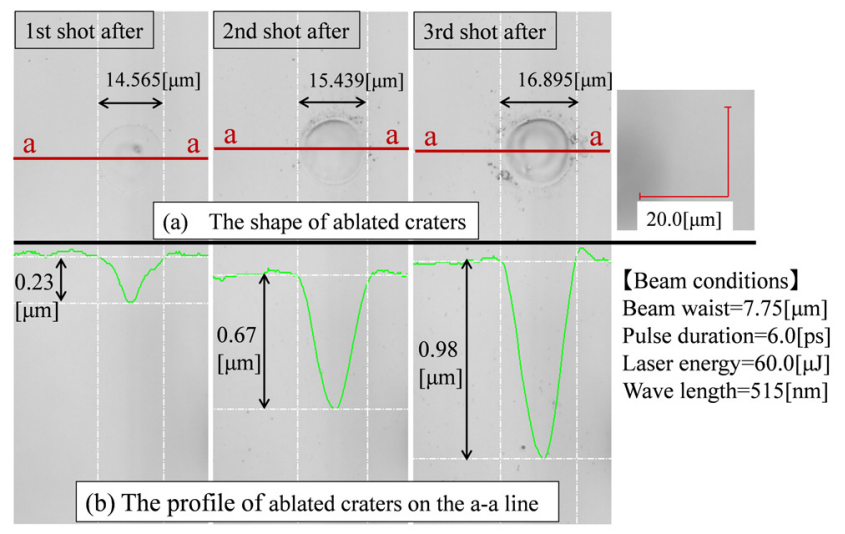

Fig. 10. The shape of ablated craters and the profile of ablated craters on the a-a line.

the linearity of the ablation rate in the second shot and in subsequent shots was accepted, but it was overestimated when beam waist was large. The gap between the analytical and experimental results until the second shot is remarkable when attention is paid to the ablated crater depth of the beam waist: $W_{0}=11.50 \mu \mathrm{m}$. As this result, it is expected that the modification of threshold fluence is related to not only the number of shots but also to the distance from the surface of the sample. Since it is so efficient in actual micro drilling processing that there are high ablation rates, after taking into consideration the laser fluence dependence of the absorption coefficient and the threshold fluence in initial shots, we think that the smallest possible beam waist and higher laser energies should be used.

The ablated crater shape is acquired in the analytical model. The variation in the ablated crater shape up to five shots is shown in Fig. 9 under the calculation conditions presented in Table 1 for three beam waists, $W_{0}=7.75$, 11.50 and $15.75 \mu \mathrm{m}$. The ablated crater depth shown in Fig. 9 is a value of the deepest part directly under the peak laser intensity of Fig. 8.

Figure 10 presents the measurements of ablated crater shapes for up to three shots to $Q=60.0[\mu \mathrm{J}], W_{0}=7.75$ $[\mu \mathrm{m}]$. Fig. 10(a) is the shape of ablated craters and Fig. 10(b) is the surface profile of ablated craters on the 
a-a line, as shown in Fig. 10(a). The surface profile after a second shot became a cone, and analytical and experimental results were largely in agreement. On the other hand, in single-shot, the crater took on a cone shape in experimental results but the parabolic shape in analytical results and a difference was found. However, the difference of ablated crater shape in analytical and experimental results was not particularly noticeable in the deep region more than $0.5 \mu \mathrm{m}$.

\section{Conclusion}

The purpose of this study has been to estimate ablated crater depth with sufficient numerical accuracy when multi-shot channels of ultra-short pulsed laser are executed for micro drilling processes on glass plates. In this analytical model, the plasma model, in which the free electron density is evaluated and the complex dielectric function of the Lorentz model is applied to evaluate ablated regions and regions damaged by laser ablation when glass is considered as dielectric material.

The following conclusions were reached.

1) Threshold fluence, which was in good agreement with experimental results, could be computed quantitatively for several laser irradiation conditions.

2) Absorption coefficient depended on laser fluence. The absorption coefficient increased as laser fluence rose. It is suspected that the reduction in ablated crater depth was caused by the increment of the absorption coefficient.

3) When absorption coefficients and threshold fluences obtained from this analytical model were applied to the Beer-Lambert law, ablation rates evaluated by the Beer-Lambert law were in good agreement with those evaluated using this analytical model over a wide range of laser fluences.

4) In laser ablation experiments involving irradiation by multi-shot laser beams, ablation rates for the initial shots were lower than subsequent ablation rates. The effectiveness of the modified absorption coefficient and modified threshold fluence of the initial shots was confirmed for the reduction of the ablation rate.

\section{Acknowledgements}

The authors would like to extend thanks to TRUMPF Corporation for the ultra-short pulsed laser frequency devices used in this study. This study is based on projects supported by Fraunhofer Institute for Laser technology (ILT) in Germany and TRUMPF Corporation in Japan. Their support is gratefully acknowledged.

\section{References:}

[1] J. R. Vazquez de Aldana, C. Mendez, L. Roso, and P. Moreno, "Propagation of ablation channels with multiple femtosecond laser pulses in dielectrics: numerical simulations and experiments," J. of Phys. D: Applied Physics, Vol.38, pp. 2764-2768, 2005.
[2] M. Sun, U. Eppelt, S. Russ, C. Hartmann, C. Siebert, J. Zhu, and W. Schulz, "Numerical analysis of ablation and damage in glass with multiple picosecond laser pulses," Optics Express, Vol.21, No.7, pp. 7858-7868, 2013.

[3] L. Jiang and H. L. Tsai, "A plasma model combination with improved two-temperature equation for ultrafast ablation of dielectrics," J. of Applied Physics, Vol.104, pp. 093101-1-8, 2008.

[4] L. Jiang and H. L. Tsai, "Energy transport and material removal in wide bandgap materials by a femtosecond laser pulse," I. J. Heat and Mass Transfer, Vol.48, pp. 487-499, 2005.

[5] L. Jiang and H. L. Tsai, "Improved Two-emperature Model and Its Application in Ultrashort Laser Heating of Metal Films," J. Heat Transfer, Vol.127, pp. 1167-1173, 2005.

[6] A. Ben-Yakar and R. L. Byer, "Femtosecond laser ablation properties of borosilicate glass," J. of Applied Physics, Vol.96, No.9, pp. 5316-5323, 2004.

[7] A. Ben-Yakar, A. Harkin, J. Ashmore, R. L. Byer, and H. A. Stone, "Thermal and fluid processes of a thin melt zone during femtosecond laser ablation of glass: the formation of rims by single laser pulses," J. of Phys. D: Applied Physics, Vol.40, pp. 1447-1459, 2007.

[8] P. K. Kennedy, "A First-Order Model for Computation of LaserInduced Breakdown Thresholds in Ocular and Aqueous Media: Part I-Theory," IEEE J. of Quantum Electronics, Vol.31, No.12, pp. 2241-2249, 1995.

[9] C. H. Fan, J. Sun and J. P. Longtin, "I Breakdown threshold and localized electron density in water induced by ultrashort laser pulses," J. Applied Physics, Vol.91, No.4, pp. 2530-2536, 2002.

[10] V. S. Popov, "Tunnel and multiphoton ionization of atoms and iones in a strong laser field (Keldysh theory)," Physics Uspekhi, Vol.47, No.9, pp. 855-885, 2004.

[11] L. V. Keldysh, "Ionization in the field of a strong electromagnetic wave," Soviet Physics JETP, Vol.20, No.5, pp. 1307-1314, 1965.

[12] A. Kaiser, B. Rethfeld, M. Vicanek, and G. Simon, "Microscopic processes in dielectrics under iradiation by subpicosecond laser pulses," Physical review B, Vol.61, No.17, pp. 437-450, 2000.

[13] B. C. Stuart, M. D. Feit, S. Herman, A. M. Rubenchik, B. W. Shore, and M. D. Petty, "Nanosecond-to-femtosecond laser-induced breakdown in dielectrics," Physical review B, Vol.53, No.4, pp. 17491761, 1996.

[14] M. Lenzner, J. Kruger, S. Sartania, Z. Cheng, C. Spielmann, G. Mourou, W. Kautec, and F. Krausz, "Femtosecond optical breakdown in dielectrics," Physical review letters, Vol.80, No.18, pp. 4076-4079, 1998.

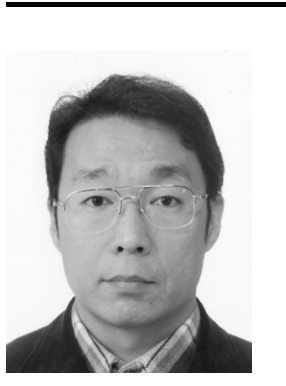

Name:

Fumitaka Motomura

\section{Affiliation:}

Assistant Lecturer, Human and Engineered Environmental Science, Division of System Science, Nagasaki University

Address:

1-14 Bunkyo-machi, Nagasaki-shi, Nagasaki 852-8521, Japan

Brief Biographical History:

1995- Nagasaki University

Main Works:

- "In-Situ Observation of Propagating-Crack Front Geometry in Thermal Stress Cleaving of Thin Glass Plate," JSME Series A, Vol.75-755, pp. 925-932, 2009.

Membership in Academic Societies:

- Japan Laser Processing Society (JLPS)

- Japan Society of Mechanical Engineers (JSME)

- Japan Society of Precision Engineering (JSPE) 